(2) Open Access Full Text Article

\title{
Hypertriglyceridemia as an Independent Predictor for Ten-Year Incidence of Diabetes in Thais
}

\author{
Suranut Charoensri (iD) \\ Supatida Turnsaket ${ }^{2}$ \\ Chatlert Pongchaiyakul' \\ 'Division of Endocrinology and \\ Metabolism, Department of Medicine, \\ Faculty of Medicine, Khon Kaen \\ University, Khon Kaen, Thailand; \\ ${ }^{2}$ Department of Medicine, Faculty of \\ Medicine, Khon Kaen University, Khon \\ Kaen, Thailand
}

Background: Fasting hypertriglyceridemia commonly associates with insulin resistance and is frequently prevalent in type 2 diabetes mellitus (DM). However, hypertriglyceridemia has not been investigated as an independent predictor of incidence of DM, especially in Thais. Methods: A 10-year hospital-based retrospective cohort study was conducted in a tertiary care setting in Thailand. Health check-up data in 2007 from healthy participants without underlying disease were extracted as baseline data. In 2017, 10 years following an initial examination, the diagnosis of DM and other laboratory data were identified. Hypertriglyceridemia was defined as fasting triglyceride level $\geq 150 \mathrm{mg} / \mathrm{dL}$. A generalized additive model (GAM) was applied to demonstrate a relationship between fasting TG level and probability of incident DM in 10 years. An association between hypertriglyceridemia and 10-year incidence of DM was evaluated using univariable and multivariable logistic regression analysis.

Results: A total of 1342 non-diabetic adults with complete both baseline and 10-year follow-up data were included in the analysis. The incidence of DM in the study period was $10.3 \%$. Baseline fasting triglyceride level is significantly higher in participants with incidence of DM, with a median difference of $45 \mathrm{mg} / \mathrm{dL}(P<0.01)$. Univariable logistic regression showed that hypertriglyceridemia was associated with 10-year incidence of DM (odds ratio (OR) 3.03, 95\% CI 2.12-4.35). After adjusting for potential confounders, hypertriglyceridemia remained significantly associated with incidence of DM (OR 2.33, 95\% CI 1.61-3.39).

Conclusion: Fasting triglyceride level is an independent risk factor for the development of new-onset DM. Testing for hypertriglyceridemia in people without diabetes may be an alternative screening tool to identify populations at risk of developing future DM, as well as providing triglyceride as a new target for DM risk reduction.

Keywords: hypertriglyceridemia, predictor, diabetes, incidence

\section{Introduction}

Diabetes mellitus (DM), especially type 2, is a major public health problem that is reaching epidemic status worldwide. ${ }^{1}$ According to the Thai National Health Examination Survey in 2016, 4.8 million people in Thailand are living with type $2 \mathrm{DM}^{2}$ Chronic hyperglycemia in people with DM is associated with long-term vascular complications, especially retinopathy, nephropathy, and cardiovascular diseases. ${ }^{3,4}$ As a chronic disease, DM has an enormous economic and psychosocial burden. Preventive strategies are needed to decrease the incidence of DM, particularly in individuals who have higher risks for DM.

Fasting hypertriglyceridemia, which is a common metabolic feature in Asians (9.0-38.6\%, according to the definitions), ${ }^{5}$ has emerged as a risk factor for type 2
Correspondence: Chatlert Pongchaiyakul Division of Endocrinology and

Metabolism, Department of Medicine, Faculty of Medicine, Khon Kaen University, Khon Kaen, 40002, Thailand

Tel +66-43-363664

Email pchatl@kku.ac.th 
DM. Elevated fasting triglyceride (TG) levels commonly associate with insulin resistance and are frequently prevalent in type $2 \mathrm{DM}^{6}{ }^{6}$ A fasting TG level of $150 \mathrm{mg} / \mathrm{dL}$ or more is one of the criteria for defining the syndrome of insulin-resistant state called "metabolic syndrome". Although this relationship between TG and insulin resistance has long been recognized, the causal relationship of these two entities is still a matter of debate. Several studies have reported that elevated TG is an independent risk factor for the future development of type $2 \mathrm{DM}^{8-11}$ Some studies found that elevated TG may occur more than 10 years before the onset of newly diagnosed DM. ${ }^{12,13}$ However, fasting TG levels have not been investigated as an independent predictor of incidence of DM after adjusting for potential confounders, especially in Thais. Therefore, this study aimed to explore whether fasting TG levels in healthy normoglycemic adults are associated with future incidence of DM in Thailand.

\section{Materials and Methods}

\section{Study Design and Participants}

A 10-year hospital-based retrospective cohort study was conducted at Srinagarind Hospital, a tertiary care setting in Thailand. Health check-up data, which consisted of a completed medical history and laboratory results of 4359 adult participants (age $\geq 18$ years) in 2007, were extracted and defined as the "baseline" dataset. The baseline dataset included sex, age, body mass index (BMI), blood pressure, and the fasting level from a single measurement of plasma glucose, serum creatinine, total cholesterol (TC), and TG. Another health check-up dataset in 2017, 10 years later, was extracted and defined as the "10year follow-up" dataset. All hospital numbers (HN) of every participant in a 10-year follow-up dataset were matched with the baseline dataset, resulting in a total of 1754 participants with complete information at both baseline and 10-year follow-up. Subjects who had been previously diagnosed with DM before participating in the baseline health check-up or had initial laboratory results consistent with the diagnosis of DM were excluded from the study. Subjects with a history of taking any medications which could affect the metabolic profiles (including glucocorticoids, contraceptive pills, neuropsychiatric agents, statins, fibrates, and diuretics) were also excluded. After exclusion, 1342 participants remained in the final analysis. The Human Research Ethics Committee of Khon Kaen University reviewed and approved the study per the
Helsinki Declaration and the Good Clinical Practice Guidelines (HE611278). Because the study was a secondary analysis of existing data, the need for obtaining informed consent was waived. Non-identified information was used in order to protect the patient data confidentiality.

\section{Definition}

According to 2010 American Diabetes Association (ADA) criteria, ${ }^{14} \mathrm{DM}$ was defined in subjects as fasting plasma glucose (FPG) $\geq 126 \mathrm{mg} / \mathrm{dL}$ and/or if the subject is currently using antidiabetic medication. Hypertriglyceridemia and hypercholesterolemia was defined as TG of $150 \mathrm{mg} /$ $\mathrm{dL}$ or more and TC of $200 \mathrm{mg} / \mathrm{dL}$ or more, ${ }^{15}$ respectively. Hypertension (HT) was classified as systolic blood pressure (SBP) of more than $140 \mathrm{mmHg}$ and/or diastolic blood pressure (DBP) of more than $90 \mathrm{mmHg} .{ }^{16}$ Mean arterial blood pressure (MAP) was calculated by the summary of SBP and 2 folds of DBP divided by 3. Chronic kidney disease (CKD) was defined according to estimated glomerular filtration rate (eGFR) of $60 \mathrm{~mL} / \mathrm{min}$ or less. ${ }^{17}$ The eGFR was calculated by the Chronic Kidney Disease Epidemiology Collaboration (CKD-EPI) equation. ${ }^{18}$ Subjects with BMI of $25 \mathrm{~kg} / \mathrm{m}^{2}$ or more were classified as obese. BMI was calculated by dividing weight $(\mathrm{kg})$ by height ${ }^{2}\left(\mathrm{~m}^{2}\right)$.

\section{Incident DM}

Incident DM was examined by reviewing all the hospital outpatient records and electronic medical records. The diagnosis of DM, which had been confirmed according to 2010 ADA criteria, ${ }^{14}$ at any time during the 10 -year follow-up period, is identified as an outcome of the study. An association between TG level at the first clinical assessment and 10-year incidence of DM was evaluated.

\section{Statistical Analysis}

All statistical analyses were performed using $\mathrm{R}$ (de Micheaux, Drouilhet, and Liquet, 2014). Data were presented as median and interquartile range (IQR 25-75\%) and proportions for continuous and categorical variables, respectively. Wilcoxon rank sum test, chi-square or Fisher's exact test was used to calculate the $P$-value as appropriate. A generalized additive model (GAM) was applied to demonstrate a relationship between fasting TG level and probability of incident DM in 10 years. Univariable logistic regression analysis was performed to calculate the odds ratios (ORs) and 95\% confidence 
Table I Characteristics of Study Participants According to the Diagnosis of DM at the End of I0-Year Follow-Up

\begin{tabular}{|c|c|c|c|c|}
\hline & Total $(n=1342)$ & No DM $(n=1204)$ & $D M(n=138)$ & $P$-value \\
\hline Male, $n(\%)$ & $452(33.7)$ & $396(32.9)$ & $56(40.6)$ & 0.07 \\
\hline Age, years & $46.0(41.0,53.8)$ & $46.0(41.0,53.0)$ & $52.0(44.0,59.0)$ & $<0.00 I^{*}$ \\
\hline BMI, $\mathrm{kg} / \mathrm{m}^{2}$ & $23.7(21.6,26.0)$ & $23.4(21.4,25.7)$ & $26.2(23.8,28.1)$ & $<0.00 I^{*}$ \\
\hline SBP, mmHg & $116(110,130)$ & $110(110,123)$ & $120(110,130)$ & $<0.00 I^{*}$ \\
\hline $\mathrm{DBP}, \mathrm{mmHg}$ & $80(70,80)$ & $80(70,80)$ & $80(70,90)$ & $<0.00 I^{*}$ \\
\hline $\mathrm{FPG}, \mathrm{mg} / \mathrm{dL}$ & $86.0(79.0,93.0)$ & $85.0(79.0,92.0)$ & $97.0(89.0,105.5)$ & $<0.001^{*}$ \\
\hline eGFR, $\mathrm{mL} / \mathrm{min}$ & $94.5(80.6,106.5)$ & $95.1(80.9,106.5)$ & $91.1(77.9,104.4)$ & $0.04 *$ \\
\hline Cholesterol, mg/dL & $208.0(182.0,235.0)$ & $207.0(181.0,234.5)$ & $215.0(189.3,238.0)$ & 0.06 \\
\hline Triglyceride, mg/dL & $105.0(72.0,153.8)$ & $101.0(70.0,148.0)$ & $146.0(100.3,221.8)$ & $<0.001^{*}$ \\
\hline Obesity, $n(\%)$ & $459(34.2)$ & $377(31.3)$ & $82(59.4)$ & $<0.00 I^{*}$ \\
\hline Hypertension, $n$ (\%) & $328(24.4)$ & $278(23.1)$ & $50(36.2)$ & $<0.001^{*}$ \\
\hline CKD, $n(\%)$ & $46(3.4)$ & $41(3.4)$ & $5(3.6)$ & 0.80 \\
\hline Hypercholesterolemia, $n$ (\%) & $78 \mid(58.2)$ & 691 (57.4) & $90(65.2)$ & 0.08 \\
\hline Hypertriglyceridemia, $n$ (\%) & $360(26.8)$ & $292(24.3)$ & $68(49.3)$ & $<0.01 *$ \\
\hline
\end{tabular}

Notes: Data are presented as median and interquartile range (IQR) and proportions for continuous and categorical variables, respectively. Wilcoxon rank-sum test and chisquare were used to calculate the $P$-value as appropriate. ${ }^{* P}$-value $<0.05$ was considered statistically significant.

Abbreviations: DM, diabetes mellitus; BMI, body mass index; SBP, systolic blood pressure; DBP, diastolic blood pressure; FPG, fasting plasma glucose; eGFR, estimated glomerular filtration rate; CKD, chronic kidney disease.

interval $(95 \% \mathrm{CI})$ between triglyceride category levels and 10 -year incidence of DM. Multivariable logistic regression was used to assess confounding and effect modification from other potential risks. $P$-value $<0.05$ was considered statistically significant.

\section{Results}

Overall, 1342 subjects (452 men and 890 women) were included in the final analysis. Incident DM during 10-year follow-up was diagnosed in 138 subjects (10.3\%). The baseline characteristics of participants who developed incident DM were compared to those with those without incident DM. No gender difference was observed between the two groups. The median age (IQR) of the total population was 46.0 (41.0, 53.8) years. Participants who developed incident DM were significantly older, had higher BMI, SBP, and DBP, but lower eGFR. However, TC was not different. Baseline fasting TG level was significantly higher in participants with incident DM, with a median difference of $45 \mathrm{mg} / \mathrm{dL}$. The subjects who developed incident DM had higher prevalence of obesity, HT, and hypertriglyceridemia at baseline (Table 1). The initial TG levels stratified by gender and incidence of DM during 10-year follow-up are shown (Figure 1). Subjects with incident DM had higher fasting TG level in both men and women $(P$-value $<0.05)$. A generalized additive model showed a positive correlation between fasting TG level and the probability of incident DM in 10 years $(P$-value $<0.001)$. The probability of DM plateaued at around $70 \%$ at very high levels of TG (Figure 2).
In univariable logistic regression analysis, fasting TG in the hypertriglyceridemic range ( $\mathrm{TG} \geq 150 \mathrm{mg} / \mathrm{dL}$ ) was associated with 10-year incidence of DM during 10-year followup, with odds ratio (OR) of 3.03 (95\% CI, 2.12-4.35). Every category level of fasting TG elevation was associated with incidence of DM. Multivariable logistic regression analysis was performed to assess confounders. After adjusting for sex, age, BMI, SBP, DBP, eGFR, and FPG at baseline, we found that hypertriglyceridemia remained as a significant predictor of incident DM. ORs when compared with fasting $\mathrm{TG}<100 \mathrm{mg} / \mathrm{dL}$ were 1.14 (95\% CI, 1.15-3.92), 3.00 (95\% CI, 1.47-6.01), and 3.54 (95\% CI, 1.80-6.88) for fasting TG 150-199, 200-249, and $\geq 250 \mathrm{mg} / \mathrm{dL}$, respectively (Table 2). Moreover, subgroup analyses indicated that the association between hypertriglyceridemia and DM was more predominant in men, those who were older than 60 years, and those who have higher BMI. However, no interaction was found between subgroup variables (Table 3 ).

\section{Discussion}

Our findings revealed that fasting hypertriglyceridemia was an independent predictor of incident DM. A higher TG level predicted higher odds of the occurrence of DM in 10-year follow-up, regardless of age, gender, BMI, blood pressure, renal function, and baseline fasting plasma glucose. We also demonstrated the correlation between fasting TG level and the probability of 10-year incidence of DM in a generalized additive model, which found that the probability of incident DM in 10 years had a positive correlation with an increased 


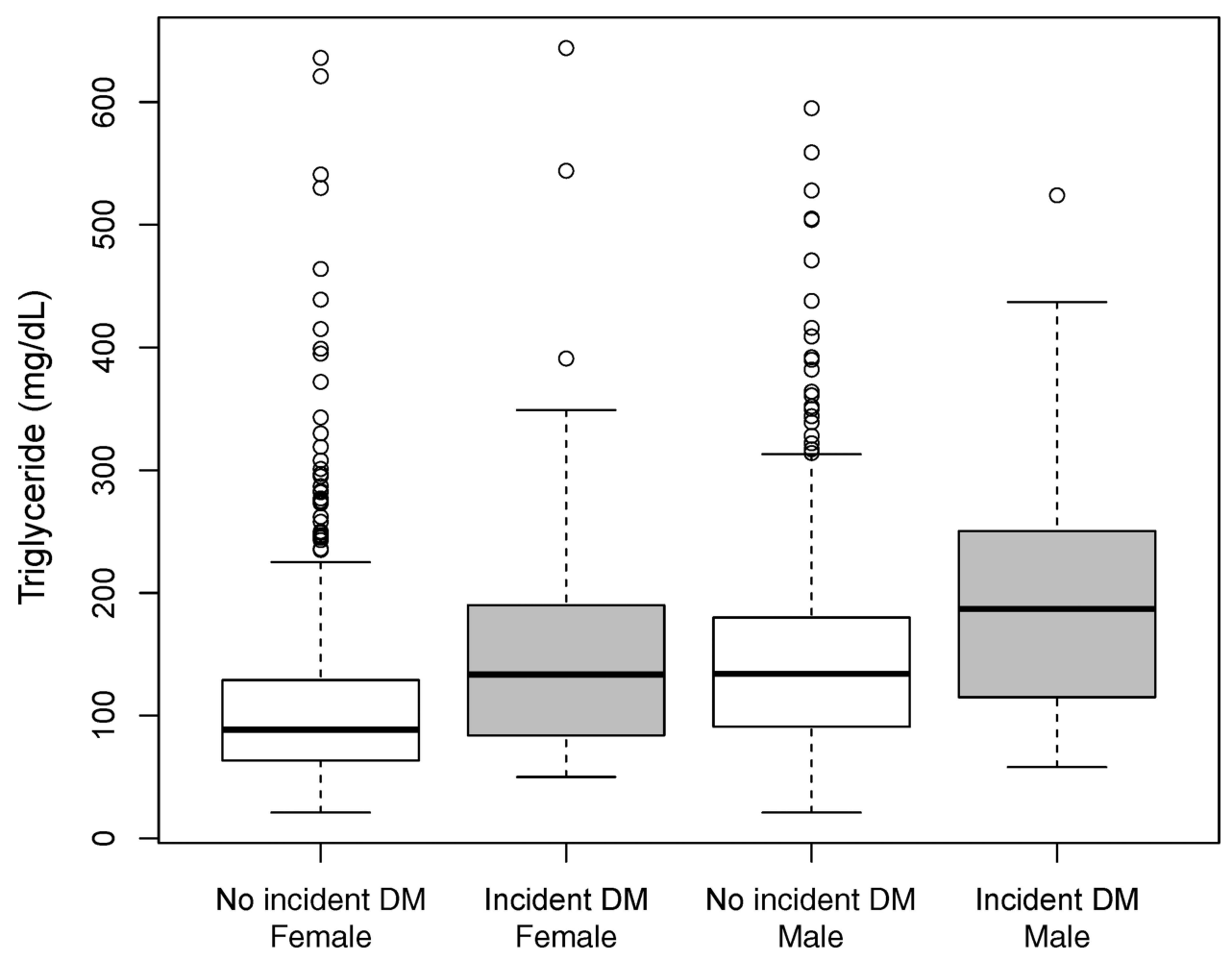

Figure I Baseline triglyceride levels stratified by sex and incident diabetes after 10-year follow-up.

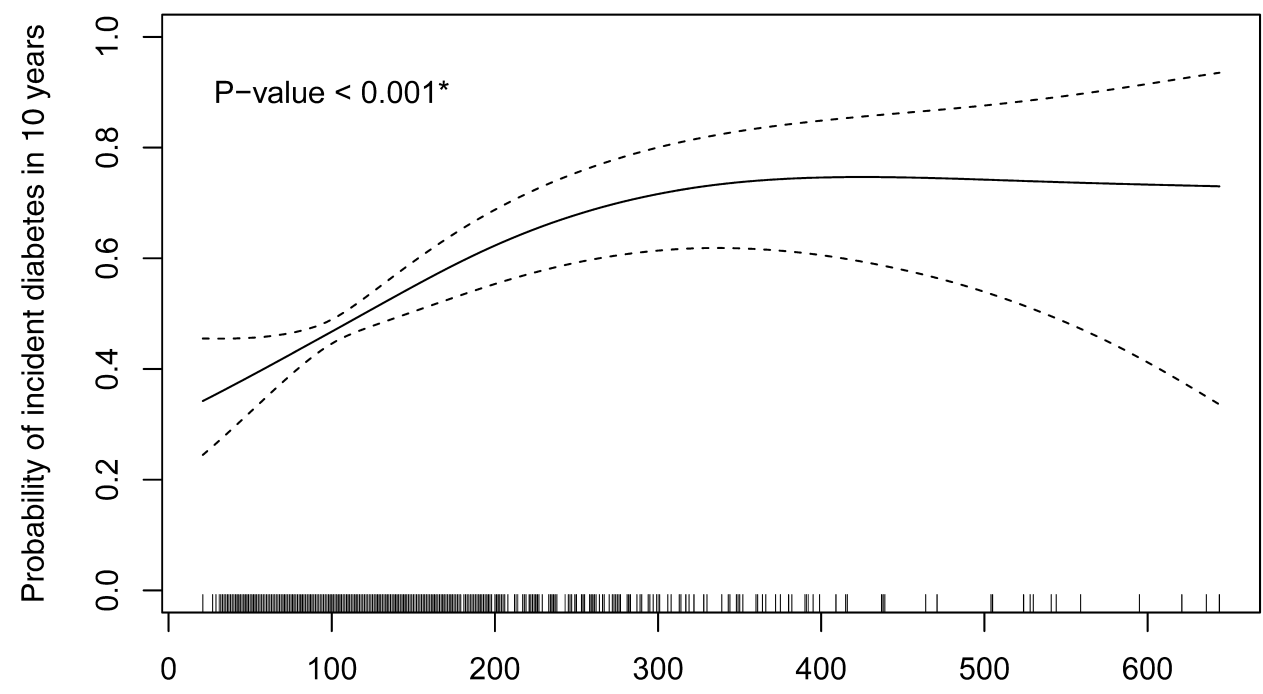

Triglyceride (mg/dL)

Figure 2 A correlation between fasting TG level and probability of incident DM in 10 years. Smoothed curve of the additive effect to the estimated probability of incident DM in 10 years for the triglyceride levels in the generalized additive model (GAM). Dashed lines represent $95 \%$ confidence intervals, marks along the lower axis represent a single observation. A straight line represents an additive effect of zero. $* P$-value $<0.05$ was considered statistically significant.

TG level, even when the rising TG level remained within the normal range. These results support the hypothesis that triglycerides are a risk factor for worsening insulin resistance and type $2 \mathrm{DM}$, which have been postulated in numerous
Western studies. ${ }^{8,9,12,13}$ In Asian studies, our result was also consistent with some earlier reports which revealed that TG level might be an influential factor for type $2 \mathrm{DM}$ in Korean, ${ }^{19}$ Chinese, ${ }^{11,20}$ and Japanese populations. $^{21}$ 
Table 2 Univariable and Multivariable Logistic Regression Analysis of Hypertriglyceridemia, Triglyceride Category Levels, and Incidence of Diabetes After 10-Year Follow-Up

\begin{tabular}{|l|c|c|c|c|}
\hline & \multicolumn{3}{|c|}{ Incidence of Diabetes After I0-Year Follow-Up } \\
\hline Triglyceride Category & Crude OR (95\% Cl) & P-value & Adjusted OR $\mathbf{~}^{\dagger} \mathbf{( 9 5 \%}$ CI) & $P_{\text {-value }}$ \\
\hline Hypertriglyceridemia & $3.03(2.12-4.35)$ & $<0.00 I^{*}$ & $2.28(1.52-3.43)$ & $<0.00 I^{*}$ \\
$<100 \mathrm{mg} / \mathrm{dL}$ & Reference & - & Reference & - \\
$100-149 \mathrm{mg} / \mathrm{dL}$ & $2.06(1.27-3.38)$ & $0.004^{*}$ & $1.39(0.80-2.39)$ & 0.24 \\
$150-199 \mathrm{mg} / \mathrm{dL}$ & $2.89(1.66-4.99)$ & $<0.00 I^{*}$ & $2.14(1.15-3.92)$ & $0.02^{*}$ \\
$200-249 \mathrm{mg} / \mathrm{dL}$ & $5.60(3.00-10.26)$ & $<0.00 I^{*}$ & $3.00(1.47-6.01)$ & $0.002^{*}$ \\
$\geq 250 \mathrm{mg} / \mathrm{dL}$ & $5.64(3.12-10.10)$ & $<0.00 I^{*}$ & $3.54(1.80-6.88)$ & $<0.00 I^{*}$ \\
\hline
\end{tabular}

Notes: Hypertriglyceridemia was defined as fasting triglyceride of $150 \mathrm{mg} / \mathrm{dL}$ or more. ${ }^{*} P$-value $<0.05$ was considered statistically significant. ${ }^{\dagger}$ Model adjusted for age, sex, body mass index, systolic blood pressure, diastolic blood pressure, estimated glomerular filtration rate, and fasting plasma glucose level.

Table 3 Multivariable Logistic Regression Analysis of Hypertriglyceridemia and Incidence of Diabetes After I0-Year Follow-Up When Categorized into Subgroups of Sex, Age, and Body Mass Index

\begin{tabular}{|c|c|c|}
\hline \multicolumn{3}{|c|}{$\begin{array}{l}\text { Hypertriglyceridemia and Incidence of Diabetes After 10- } \\
\text { Year Follow-Up }\end{array}$} \\
\hline Subgroups & $\begin{array}{l}\text { Adjusted OR† }(95 \% \\
\mathrm{Cl})\end{array}$ & $P$ for Interaction \\
\hline Sex & & 0.77 \\
\hline Male & $2.54(1.33-4.98)$ & \\
\hline Female & $2.12(1.23-3.62)$ & \\
\hline Age group & & 0.55 \\
\hline$\geq 60$ years & $3.77(1.39-10.81)$ & \\
\hline$<60$ years & $2.25(1.42-3.57)$ & \\
\hline Body mass index & & 0.54 \\
\hline (BMI) & & \\
\hline$<25 \mathrm{~kg} / \mathrm{m}^{2}$ & $2.05(1.08-3.83)$ & \\
\hline$\geq 25 \mathrm{~kg} / \mathrm{m}^{2}$ & $2.39(1.37-4.19)$ & \\
\hline
\end{tabular}

Notes: Hypertriglyceridemia was defined as fasting triglyceride of $150 \mathrm{mg} / \mathrm{dL}$ or more. 'Model adjusted for age, sex, body mass index, systolic blood pressure, diastolic blood pressure, estimated glomerular filtration rate, and fasting plasma glucose level at baseline.

A limited number of studies have been performed to evaluate the effects of lipid profiles on the incidence of DM in Thailand. To the best of our knowledge, our study is the first study in Thais to assess the relationship between triglyceride level and future DM which confirms the significance of triglyceride as a predictor of incident DM in Asian populations. Such information may help identify individuals who are at high risk of DM, as well as providing triglyceride as a new target for DM risk reduction besides BMI.

Hypertriglyceridemia has been recognized as one of the key components in metabolic syndrome, the syndrome of an insulin-resistant state which is also related with hypertension and glucose intolerance. ${ }^{7}$ However, the causal relationship between these conditions remains controversial. A recent study in Chinese populations found that TG positively correlated with increased insulin resistance and negatively correlated with beta cell function, even in individuals with normal glucose tolerance. $^{22}$ This finding confirmed our hypothesis that TG is an independent risk factor of future DM which could be attributed to the aggravation of insulin resistance and acceleration of islet beta cell dysfunction. Therefore, for patients with fasting hypertriglyceridemia, it is necessary to strengthen the control of hypertriglyceridemia even if the plasma glucose is normal. The management of hypertriglyceridemia, including lifestyle modifications and the use of drugs such as fibrates, niacin, and omega3 fatty acids, as recommended by Endocrine Society Clinical Practice Guidelines, ${ }^{23}$ should be encouraged.

Many well-established clinical risk factors of DM have been incorporated in the standard prediabetes and type 2 DM screening tool, including sex, age, and BMI. ${ }^{3}$ In our subgroup analysis of diabetic risk factors, the positive relationship of hypertriglyceridemia and incident DM remained consistent in both men and women, younger and elderly participants, lean and overweight/obese participants, with no significant interaction between subgroups. However, participants with conventional risks of DM, including male gender, age of more than 60 years, and higher BMI, tended to have a more prominent association between hypertriglyceridemia and the development of DM. These findings were consistent with recent study in China ${ }^{24}$ and suggested that triglyceride level, in addition to the other well-recognized risk factors, should be considered as an adjunctive variable in the predictive model of DM. 
The prevalence of DM in our population at the end of 10 -year follow-up was $10.3 \%$, which was slightly higher than the overall prevalence in Thailand. According to the data from the International Diabetes Federation (IDF) in 2019, diabetes prevalence in adults aged 20-79 years in Thailand was $8.3 \%{ }^{25}$ The higher prevalence of DM in our cohort could be attributed to the study setting, which was conducted in the northeastern region of Thailand. The study populations were living in a rural area and likely to be in a lower socioeconomic status than the nationally representative group, which mostly resided in Bangkok, the capital city of Thailand. A cross-sectional study in Thais using the data from the National Socioeconomics Survey in 2010 and 2012 found that those with lower socioeconomic status were more vulnerable to $\mathrm{DM},{ }^{26}$ which supports our hypothesis.

The strengths of our study include a large cohort, a long follow-up period of 10-years, and data collection in a registered health check-up database that minimized information bias. However, the present findings should be interpreted in the context of potential limitations. First, metabolic parameters which are closely related to TG, such as hemoglobin A1C (HbA1c), high-density lipoprotein cholesterol (HDL-C), and low-density lipoprotein cholesterol (LDL-C), were missing, which may affect the integrity of our results. Second, since this study was a secondary analysis from the existing data, we do not have data regarding other risk factors of DM during the study period, including dietary habits, nutritional therapy, exercise, or medications, which could act as confounders. Moreover, we do not have follow-up information of all the metabolic profiles during the 10-year follow-up duration. Since metabolic parameters such as SBP, DBP, FPG, eGFR, and lipid profiles can change over time, the lack of data regarding these changes can affect the validity of our result. Nevertheless, our study design reflects a reallife health check-up scenario in which patients attended the clinic only once, only a single clinical and laboratory assessment was made, and follow-up data were sometimes unavailable.

In conclusion, fasting triglyceride level is an independent risk factor for the development of incident DM in 10 years. Testing for hypertriglyceridemia in people without DM may be an alternative screening tool to identify the population at risk of developing future DM.

\section{Disclosure}

The authors report no conflicts of interest in this work.

\section{References}

1. International Diabetes Federation. IDF diabetes atlas. 7th ed; 2015. Available from: https://www.diabetesatlas.org/upload/resources/pre vious/files/7/IDF\%20Diabetes\%20Atlas\%207th.pdf. Accessed 23 Apr. 2021

2. Thai National Health Examination Survey V Study Group. Thai national health examination survey, NHES V. Nonthaburi, Thailand: National Health Examination Survey Office, Health System Research Institute; 2016.

3. American Diabetes Association. 2. Classification and diagnosis of diabetes: standards of medical care in diabetes-2020. Diabetes Care. 2020;43:S14-31. doi:10.2337/dc20-S002

4. Leon BM, Maddox TM. Diabetes and cardiovascular disease: epidemiology, biological mechanisms, treatment recommendations and future research. World J Diabetes. 2015;6:1246-1258. doi:10.4239/ wjd.v6.i13.1246

5. Lin CF, Chang YH, Chien SC, Lin YH, Yeh HY. Epidemiology of dyslipidemia in the asia pacific region. Int J Gerontol. 2018;12:2-6. doi:10.1016/j.ijge.2018.02.010

6. Grundy SM. Hypertriglyceridemia, insulin resistance, and the metabolic syndrome. Am J Cardiol. 1999;83:25F-29F. doi:10.1016/ S0002-9149(99)00211-8

7. Alberti KGMM, Zimmet P, Shaw J. Metabolic syndrome-a new world-wide definition. A consensus statement from the international diabetes federation. Diabet Med J Br Diabet Assoc. 2006;23:469-480. doi:10.1111/j.1464-5491.2006.01858.x

8. Hjellvik V, Sakshaug S, Strøm H. Body mass index, triglycerides, glucose, and blood pressure as predictors of type 2 diabetes in a middle-aged Norwegian cohort of men and women. Clin Epidemiol. 2012;4:213-224. doi:10.2147/CLEP.S31830

9. Dotevall A, Johansson S, Wilhelmsen L, Rosengren A. Increased levels of triglycerides, BMI and blood pressure and low physical activity increase the risk of diabetes in Swedish women. A prospective 18-year follow-up of the BEDA study. Diabet Med $J \mathrm{Br}$ Diabet Assoc. 2004;21:615-622. doi:10.1111/j.14645491.2004.01189.x

10. Tirosh A, Shai I, Bitzur R, et al. Changes in triglyceride levels over time and risk of type 2 diabetes in young men. Diabetes Care. 2008;31:2032-2037. doi:10.2337/dc08-0825

11. Zhao J, Zhang Y, Wei F, et al. Triglyceride is an independent predictor of type 2 diabetes among middle-aged and older adults: a prospective study with 8-year follow-ups in two cohorts. $J$ Transl Med. 2019;17:403. doi:10.1186/s12967-019-02156-3

12. McLaughlin T, Abbasi F, Cheal K, Chu J, Lamendola C, Reaven G. Use of metabolic markers to identify overweight individuals who are insulin resistant. Ann Intern Med. 2003;139:802-809. doi:10.7326/ 0003-4819-139-10-200311180-00007

13. Perry IJ, Wannamethee SG, Walker MK, Thomson AG, Whincup PH, Shaper AG. Prospective study of risk factors for development of non-insulin dependent diabetes in middle aged British men. $B M J$. 1995;310:560-564. doi:10.1136/bmj.310.6979.560

14. American Diabetes Association. Executive summary: standards of medical care in diabetes-2010. Diabetes Care. 2010;33(Suppl 1): S4-10.

15. Endocrine Society. Endocrine society releases guidelines on diagnosis and management of hypertriglyceridemia. Am Fam Physician. 2013;88:142-144.

16. Carretero Oscar A, Suzanne O. Essential hypertension. Circulation. 2000;101:329-335. doi:10.1161/01.CIR.101.3.329

17. Stevens PE, Levin A; Kidney Disease: improving Global Outcomes Chronic Kidney Disease Guideline Development Work Group Members. Evaluation and management of chronic kidney disease: synopsis of the kidney disease: improving global outcomes 2012 clinical practice guideline. Ann Intern Med. 2013;158:825-830. doi:10.7326/0003-4819-158-11-201306040-00007 
18. Levey AS, Stevens LA, Schmid $\mathrm{CH}$, et al. A new equation to estimate glomerular filtration rate. Ann Intern Med. 2009;150:604-612. doi:10.7326/0003-4819-150-9-200905050-00006

19. Kwon YH, Kim SK, Cho JH, et al. The association between persistent hypertriglyceridemia and the risk of diabetes development: the kangbuk samsung health study. Endocrinol Metab Seoul Korea. 2018;33:55-61. doi:10.3803/EnM.2018.33.1.55

20. Cui J, Sun J, Wang W, et al. The association of triglycerides and total cholesterol concentrations with newly diagnosed diabetes in adults in China. Oncotarget. 2017;8:103477-103485. doi:10.18632/oncotarget. 21969

21. Kametani T, Koshida H, Nagaoka T, Miyakoshi H. Hypertriglyceridemia is an independent risk factor for development of impaired fasting glucose and diabetes mellitus: a 9-year longitudinal study in Japanese. Intern Med Tokyo Jpn. 2002;41:516-521. doi:10.2169/internalmedicine.41.516

22. Ma M, Liu H, Yu J, et al. Triglyceride is independently correlated with insulin resistance and islet beta cell function: a study in population with different glucose and lipid metabolism states. Lipids Health Dis. 2020;19:121. doi:10.1186/s12944-020-01303-w
23. Berglund L, Brunzell JD, Goldberg AC, et al. Evaluation and treatment of hypertriglyceridemia: an endocrine society clinical practice guideline. $J$ Clin Endocrinol Metab. 2012;97:2969-2989. doi:10.1210/jc.2011-3213

24. Chen C-L, Liu L, Lo K, et al. Association between triglyceride glucose index and risk of new-onset diabetes among chinese adults: findings from the china health and retirement longitudinal study. Front Cardiovasc Med. 2020;7:610322. doi:10.3389/fcvm.2020.610322

25. Saeedi P, Petersohn I, Salpea P, et al. Global and regional diabetes prevalence estimates for 2019 and projections for 2030 and 2045 results from the International Diabetes Federation Diabetes Atlas, 9th edition. Diabetes Research and Clinical Practice. 2019;157:107843

26. Suwannaphant K, Laohasiriwong W, Puttanapong N, Saengsuwan J, Phajan T. Association between socioeconomic status and diabetes mellitus: the national socioeconomics survey, 2010 and 2012 J Clin Diagn Res JCDR. 2017;11:LC18-22.

\section{Publish your work in this journal}

Vascular Health and Risk Management is an international, peerreviewed journal of therapeutics and risk management, focusing on concise rapid reporting of clinical studies on the processes involved in the maintenance of vascular health; the monitoring, prevention and treatment of vascular disease and its sequelae; and the involvement of metabolic disorders, particularly diabetes. This journal is indexed on PubMed Central and MedLine. The manuscript management system is completely online and includes a very quick and fair peerreview system, which is all easy to use. Visit http://www.dovepress. com/testimonials.php to read real quotes from published authors. 